\title{
U-shaped relationship between iodine status and thyroid autoimmunity risk in adults
}

\section{Bin Wang', Weiwei He², Qian Li', Xi Jia', Qiuming Yao', Ronghua Song³, Qiu Qin ${ }^{3}$ and Jin-an Zhang ${ }^{3}$}

'Department of Endocrinology \& Rheumatology, Jinshan Hospital of Fudan University, Shanghai, China, ${ }^{2}$ Department of Endocrinology, Affiliated Hospital of Yanan University, Shanxi, China, and ${ }^{3}$ Department of Endocrinology \& Rheumatology, Shanghai University of Medicine \& Health Sciences Affiliated Zhoupu Hospital, Shanghai, China
Correspondence

should be addressed

to J Zhang

Email

zhangjinan@hotmail.com

\begin{abstract}
Background: lodine status has long been regarded as an environmental determinant for thyroid dysfunction, but its relationship with thyroid autoimmunity (TAI) is still controversial. Our study aimed to elucidate the relationship between iodine status and TAl through both a population-based study and a dose-response meta-analysis of eligible epidemiological studies.

Methods: A population-based, cross-sectional study was firstly carried out, which enrolled a total of 2808 Chinese adults. Odds ratio (OR) with $95 \%$ confidence interval $(95 \% \mathrm{Cl})$ was calculated through logistic regression analysis. A dose-response meta-analysis of eligible epidemiological studies was also carried out.

Results: The cross-sectional study showed an U-shaped relationship between iodine intake and TAI in adults. Compared with those with more than adequate iodine status, individuals with iodine deficiency, adequate iodine status and iodine excess all had higher risk of TAl, and the adjusted ORs were $1.50(95 \% \mathrm{Cl} 1.03-2.17, P=0.032), 1.50(95 \% \mathrm{Cl}$ $1.09-2.07, P=0.013)$ and $1.68(95 \% \mathrm{Cl} 1.11-2.53, P=0.014)$, respectively. The dose-response meta-analysis included 22 epidemiological studies with a total of 69,987 participants and further validated the U-shaped relationship between iodine intake and TAI in adults, which proved the significantly increased risk of TAI among individuals with either iodine deficiency or iodine excess. Stratified analysis of studies with low risk of confounding bias also identified similar findings.

Conclusion: The study suggests an U-shaped relationship between iodine intake and TAI in adults, and both iodine deficiency and iodine excess are risk factors of TAl in adults. The underlying mechanisms need to be elucidated in future studies.
\end{abstract}

\section{Introduction}

Autoimmune thyroid diseases (AITDs) are autoimmune diseases occurring in the endocrine system and mainly include Hashimoto's thyroiditis (HT) and Graves' disease (GD) (1). Individuals with thyroid antibodies positivity are usually considered to suffer from thyroid autoimmunity (TAI) and its prevalence is over $10 \%$ in most populations $(2,3,4)$. HT is the major type of TAI and the main cause of hypothyroidism, which is also the most common autoimmune disease. TAI can result (c) 2019 European Society of Endocrinology Printed in Great Britain in thyroid dysfunction, and it also increases the risks of other common diseases, such as cardiovascular diseases, thyroid cancer and other autoimmune diseases $(5,6,7$, $8,9,10)$. Additionally, TAI is also associated with higher rates of complications during and after pregnancy, such as miscarriage and premature delivery $(3,11)$. Through numerous studies on TAI in the past two decades, it has been well accepted that TAI results from the interplay of multiple factors including susceptibility genetic variants 
and environmental factors (12). Additionally, some factors have been identified as risk factors for TAI (13, 14), but its etiology is still largely not elucidated. Though most patients are asymptomatic, TAI can still cause much adverse impact on health. Therefore, it is essential to identify those important risk factors for TAI, especially for those modifiable risk factors. Some dietary factors have been identified as risk factors for TAI and are implicated in the development of TAI, such as selenium deficiency and vitamin $\mathrm{D}$ deficiency $(15,16)$.

Iodine is essential for the synthesis of thyroid hormone and iodine status has long been regarded as a key determinant of thyroid dysfunction (17). Optimization of iodine intake in the population is critical to reduce the prevalence of thyroid diseases (17). Previous studies have established that iodine deficiency is an important risk factor for both hypothyroidism and thyroid goiter (18). Despite numerous epidemiological studies on the impact of iodine intake on thyroid dysfunction, there are limited studies assessing the impact of iodine intake on TAI. Several epidemiological studies found that excessive iodine intake could increase the risk of TAI. A randomized controlled trial by Kahaly et al. also supported that iodine could induce TAI in patients with endemic goiter (19). However, other studies reported no obvious relationship between iodine status and TAI. Therefore, the link between iodine status and TAI is complex, and convincing evidence for the association of iodine status with TAI is still lacking (17). Therefore, to adequately elucidate the relationship between iodine status and TAI risk, we conducted both a cross-sectional study and a dose-response meta-analysis.

\section{Methods}

\section{Participants}

We first performed a population-based cross-sectional study. We reported the cross-sectional study according to the Strengthening the Reporting of Observational Studies in Epidemiology (STROBE) statement (20). In the cross-sectional study, 2824 individuals were randomly recruited from residents of Jinshan District of Shanghai in 2016, and 16 were excluded from this analysis for thyroidectomy. Finally, 2808 adults were enrolled, and all were from the Chinese Han population. All participants provided written informed consent in accordance with the Declaration of Helsinki. The study was approved by the Ethics Committee of Jinshan Hospital of Fudan University.

\section{Clinical examination and laboratory testing}

Data on age, gender, smoking, disease history and use of medications were collected. In the cross-sectional study, smoking individuals were defined as those current smokers. Fasting blood was used for laboratory testing. Thyroid antibodies including thyroid peroxidase antibody (TPOAb) and thyroglobulin antibody (TGAb) were tested using immunochemiluminometric assay (ICMA) method. The reference values for TPOAb and TGAb were 0-34 IU/L and $0-50 \mathrm{IU} / \mathrm{L}$, respectively.

\section{Category criteria for iodine status}

Urinary iodine concentration (UIC) is a well-known indicator of iodine intake and is currently the most useful biochemical marker of iodine status. In our study, we also used UIC to evaluate iodine nutrition. UIC was quantified using the ammonium persulfate method. According to UIC values, participants were divided into four groups: iodine deficiency (UIC $<100 \mu \mathrm{g} / \mathrm{L}$ ), adequate iodine status (UIC 100-199.9 $\mu \mathrm{g} / \mathrm{L}$ ), more than adequate iodine status (UIC 200-299.9 $\mu \mathrm{g} / \mathrm{L}$ ) and iodine excess status (UIC $\geq 300 \mu \mathrm{g} / \mathrm{L})(21)$.

\section{Diagnostic criterion for TAI}

The outcome of interest was the prevalence of TAI in individuals with specific iodine status. TAI was referred to the presence of positive TPOAb or TGAb or a combination of these two antibodies. TPOAb positivity was defined as TPOAb $>34 \mathrm{IU} / \mathrm{L}$, and TGAb positivity was defined as $\mathrm{TGAb}>50 \mathrm{IU} / \mathrm{L}$.

\section{Statistical analysis}

We calculated sample size before the cross-sectional study. When the expected odds ratio (OR) was defined as 1.50 and the proportion with TAI in the population was $15 \%$, a sufficient power over $80 \%$ need at least 658 individuals in each group. There were four groups in our study and a total of 2632 individuals should be thus recruited. Continuous variables are presented as mean with standard deviation (S.D.) or median with interquartile range (IQR) based on their types of data distribution. Mann-Whitney $U$ test or $t$ test were used to compare the betweengroup difference. Categorical variables are presented as frequencies with percentages, and chi-square test was used to compare the difference between two groups. To uncover possible sources of bias, the difference in clinical 
characteristics across those four ordered UIC groups was also analyzed. The trend in proportions across ordered UIC groups was assessed using Cochran-Armitage test. The trend for continuous variables across ordered UIC groups was assessed using 'nptrend' in STATA. OR with 95\% confidence interval (95\% CI) was calculated through logistic regression analysis. Both age and gender had long been regarded as important impact factors of UIC and could cause risk of bias, which were thus adjusted in the logistic regression analysis. Smoking, diabetes, BMI and hypertension were also regarded as possible confounding factors in the logistic regression analysis. Age and BMI were analyzed as continuous variables, while the other factors were analyzed as categorical variables. Subgroup analyses were performed by the types of thyroid antibodies and gender. STATA (version 12.0, StataCorp, USA) was used, and two-sided $P$ values less than 0.05 were considered to be statistically significant.

\section{Dose-response meta-analysis}

To get a more comprehensive evaluation of the impact of iodine status on TAI risk, a dose-response metaanalysis was conducted in accordance with the guidance on conducting systematic reviews and meta-analyses of observational studies of etiology (COSMOS-E) (22). The research question was whether a dose-response relationship existed in the association between iodine status and TAI risk. Pubmed, EMBASE and Wanfang database were searched to identify relevant cross-sectional or cohort studies (up to 30 November 2018). No language limitation was applied. The following search strategy was used: (iodization OR iodized OR iodised OR iodine OR iodination) and (Thyroid peroxidase antibodies OR Thyroid peroxidase antibody OR Thyroglobulin antibodies OR Thyroglobulin antibody OR thyroiditis OR thyroid autoimmunity OR thyroid autoantibody OR thyroid autoantibodies). Potential studies were screened by reading title or abstract and then underwent a following full-text evaluation. The references of eligible studies were also reviewed to identify other possible studies. Studies were considered eligible if they satisfied the following inclusion criteria: (1) Using cohort or crosssectional design; (2) Participants were adults, children or adolescents; (3) Exposure was iodine status; (4) Control was those individuals with certain category of iodine status such as iodine deficiency, adequate iodine status or more than adequate iodine status; (5) The outcome of interest was TAI, TPOAb positivity or TGAb positivity; (6) Relative risk (RR) or OR with 95\% CIs for at least three quantitative categories of iodine status were reported. Reviews or studies without sufficient data were all excluded.

The following data were extracted by two investigator independently, such as the first author, year of publication, study design, number of cases, number of participants, confounding variables in the multivariable analysis, UIC testing method, definitions of TAI and risk estimates with $95 \%$ CIs in each category of iodine status. Standardized data extraction sheets were used and the discrepancy between the two investigators was solved by discussion with another investigator. Quality assessment was performed according to the Newcastle-Ottawa Quality Assessment Scale (NOS), and studies with scores no more than five points were deemed to have suboptimal quality. Moreover, the risk of bias of those included studies was further assessed by the general principles of COSMOS-E (22). Risk of confounding bias, selection bias and information bias was assessed qualitatively in three categories including 'low risk', 'moderate risk' and 'high risk' (22).

The median UIC, mean UIC or the midpoint of the upper and lower boundaries was assigned as the corresponding iodine intake for each risk estimate. If the lowest or the highest category of iodine intake was a half-open interval, their interval length was assumed to be same with that of the adjacent category. The possible nonlinear dose-response relationship between iodine status and TAI risk was assessed through restricted cubic splines, and four knots at 5, 35, 65 and 95\% of the full distribution were utilized (23). In the dose-response meta-analysis, generalized least-square regression model was used $(23,24)$. Both the spline model and the linear model were used in the dose-response meta-analysis, and the curve linearity was thus tested through the difference between the spline model and the linear model and the $P$ value for the difference above was also calculated (23). The risk estimates were pooled using a random-effect model (25). Stratified analyses by risk of confounding bias were also performed. Sensitivity analysis was then performed by excluding studies with suboptimal quality or high risk of bias. All statistical analyses were performed with STATA version 12 (StataCorp LP), and $P$ value less than 0.05 was considered to be statistically significant.

\section{Results}

\section{Characteristics of participants}

The characteristics of the 2808 participants are given in Supplementary Table 1 (see section on 
supplementary data given at the end of this article). The median UIC was $164.5 \mu \mathrm{g} / \mathrm{L}$ (IQR: 111.1-235.6). $359(12.8 \%)$ were found to have TAI, 266 (9.5\%) had TPOAb positivity and 308 (11.0\%) had TGAb positivity. Supplementary Table 1 also shows the comparison of clinical characteristics between men and women participants. Table 1 shows the difference in the clinical characteristics across those four ordered UIC groups (Table 1). As shown in Table 1, participants with high UIC had younger age, lower BMI, lower prevalence of diabetes, lower prevalence of hypertension than those with lower UIC $(P<0.05$, Table 1$)$. No obvious difference in other clinical characteristics across those four ordered UIC groups was observed (Table 1). There was no obvious difference in both the level of TPOAb $(P=0.15)$ and the level of TGAb $(P=0.19)$ across those four UIC groups (Table 1$)$. When using the adequate iodine group as reference, TPOAb level was not higher in the iodine deficiency group $(P=0.53)$, the more than adequate iodine group $(P=0.13)$ and the iodine excess group $(P=0.32)$. When using the more than adequate iodine group as reference, TPOAb level was also not higher in the iodine deficiency group $(P=0.13)$. When using the adequate iodine group as reference, TGAb level was not higher in the iodine deficiency group $(P=0.27)$, the more than adequate iodine group $(P=0.61)$ and the iodine excess group $(P=0.17)$.

\section{UIC and TAI}

The prevalence of TAI over those four ordered UIC groups is shown in Table 2, which suggested a clear U-shaped curve (Table 2). Compared with those with more than adequate iodine status (9.3\%), there was a higher prevalence of TAI in participants with iodine deficiency (14.4\%), adequate iodine status (13.5\%) and iodine excess (13.7\%) (Table 2). Multivariate logistic regression analysis also confirmed the U-shaped relationship between iodine intake and TAI (Table 2). Compared with those with more than adequate iodine status, individuals with iodine deficiency, adequate iodine status and excessive iodine status all had higher risk of TAI, and the adjusted ORs were 1.50 (95\% CI 1.03-2.17, $P=0.032), 1.50$ (95\% CI 1.09-2.07, $P=0.013$ ) and 1.68 (95\% CI 1.11-2.53, $P=0.014)$, respectively. Subgroup analysis in men also found an obvious U-shaped relationship between iodine intake and TAI (Table 2). The relationship between iodine intake and TAI in women was also likely to be U shaped, but the outcomes were not statistically significant (Table 2).

The relationship between iodine intake and TPOAb positivity was shown in Supplementary Table 2, and the relationship between iodine intake and TGAb positivity was shown in Supplementary Table 3. The relationship of iodine intake with TPOAb positivity and TGAb positivity was both $U$ shaped in men but was not in women.

\section{Dose-response meta-analysis}

Flow chart of study selection for the meta-analysis was shown in the Supplementary Fig. 1. A total of 5191 abstracts were identified through literature search. After excluding studies that did not fulfill the inclusion criteria, 185 candidate studies were further evaluated by reading full texts. Another 164 studies were further excluded and 21 studies from literature were eligible into meta-analysis $(26,27,28,29,30,31,32,33,34,35,36,37,38,39,40$, $41,42,43,44,45,46)$. Therefore, a total of 22 studies (21 studies from literature and our cross-sectional study) were finally included. Main characteristics of those studies is

Table 1 Comparison of clinical characteristics of adults with different urinary iodine concentration.

\begin{tabular}{l} 
Characteristics \\
\hline Number \\
Urinary iodine concentration \\
Age (years) \\
BMI (kg/m²) \\
Smoking $(n(\%))$ \\
Type 2 diabetes $(n(\%))$ \\
Hypertension $(n(\%))$ \\
Iodized salt intake $(n(\%))$ \\
TSH (mIU/L) \\
TPOAb $(\mathrm{U} / \mathrm{mL})$ \\
TGAb $(\mathrm{U} / \mathrm{mL})$
\end{tabular}

\begin{tabular}{|c|c|}
\hline Deficiency & Adequate \\
\hline 568 & 1253 \\
\hline $68.0(52.7-88.0)$ & $148.8(126.0-172.2)$ \\
\hline $46.5(14.4)$ & $44.5(15.6)$ \\
\hline $24.1(3.7)$ & $24.4(3.8)$ \\
\hline $113(19.9)$ & $353(28.2)$ \\
\hline $86(15.1)$ & 174 (13.9) \\
\hline $191(33.6)$ & $410(32.7)$ \\
\hline $396(69.7)$ & 955 (76.2) \\
\hline $2.7(1.8-3.2)$ & $2.7(1.7-3.2)$ \\
\hline $9.0(6.3-13.8)$ & $8.8(6.5-12.6)$ \\
\hline $11.9(10.0-15.4)$ & $11.5(10.0-15.2)$ \\
\hline
\end{tabular}

\begin{tabular}{c}
\hline More than adequate \\
\hline 616 \\
$241.5(218.7-261.9)$ \\
$41.3(16.1)$ \\
$24.0(3.8)$ \\
$185(30.0)$ \\
$70(11.4)$ \\
$174(28.2)$ \\
$513(83.3)$ \\
$2.7(1.7-3.3)$ \\
$8.6(6.3-12.2)$ \\
$11.6(10.0-14.7)$ \\
\hline
\end{tabular}

\begin{tabular}{ccc}
\hline \multicolumn{1}{c}{ Excessive } & & $\boldsymbol{P}$ \\
\cline { 1 - 1 } $652.1(332.4-1971.6)$ & & $<0.001$ \\
$39.8(17.1)$ & & $<0.001$ \\
$23.8(3.8)$ & & 0.02 \\
$101(27.2)$ & & 0.12 \\
$39(10.5)$ & & 0.01 \\
$94(25.3)$ & & 0.005 \\
$315(84.9)$ & & 0.09 \\
$2.9(1.7-3.6)$ & & 0.05 \\
$8.6(6.5-12.0)$ & & 0.15 \\
$12.4(10.0-15.4)$ & & 0.19 \\
\hline
\end{tabular}

Normally distributed variables were expressed as mean \pm S.D., non-normally distributed variables as median with IQR and categorical variables as number with percentages (\%). Urinary iodine concentrations: low, <100 $\mu$ g/L; adequate, 100-199.9 $\mu$ g/L; more than adequate, 200-299.9 $\mu$ g/L; excessive, $\geq 300 \mu \mathrm{g} / \mathrm{L}$.

BMI, body mass index; TGAb, thyroglobulin antibody; TPOAb, thyroid peroxidase antibody; TSH, thyroid-stimulating hormone. 
Table 2 Risk of thyroid autoimmunity in adults with different urinary iodine concentrations.

\begin{tabular}{|c|c|c|c|c|c|c|}
\hline \multirow[b]{2}{*}{ Urinary iodine } & \multirow[b]{2}{*}{ Number } & \multirow[b]{2}{*}{ TAI (\%) } & \multicolumn{2}{|c|}{ Crude OR } & \multicolumn{2}{|c|}{ Adjusted OR* } \\
\hline & & & OR $(95 \% \mathrm{Cl})$ & $P$ & OR $(95 \% \mathrm{Cl})$ & $P$ \\
\hline \multicolumn{7}{|l|}{ Total } \\
\hline Deficiency & 568 & $82(14.4)$ & $1.65(1.16-2.37)$ & 0.006 & $1.50(1.03-2.17)$ & 0.032 \\
\hline Adequate & 1253 & $169(13.5)$ & $1.53(1.11-2.10)$ & 0.009 & $1.50(1.09-2.07)$ & 0.013 \\
\hline More than adequate & 616 & $57(9.3)$ & 1 & - & 1 & - \\
\hline Excessive & 371 & $51(13.7)$ & $1.56(1.05-2.34)$ & 0.029 & $1.68(1.11-2.53)$ & 0.014 \\
\hline \multicolumn{7}{|l|}{ Men } \\
\hline Deficiency & 217 & $21(9.7)$ & $2.50(1.22-5.10)$ & 0.012 & $2.83(1.34-5.99)$ & 0.006 \\
\hline Adequate & 633 & $52(8.2)$ & $2.09(1.12-3.89)$ & 0.021 & $1.98(1.06-3.71)$ & 0.033 \\
\hline More than adequate & 316 & $13(4.1)$ & 1 & - & 1 & - \\
\hline Excessive & 213 & $19(8.9)$ & $2.28(1.10-4.73)$ & 0.026 & $2.31(1.11-4.83)$ & 0.026 \\
\hline \multicolumn{7}{|l|}{ Women } \\
\hline Deficiency & 351 & $61(17.4)$ & $1.22(0.80-1.87)$ & 0.349 & $1.19(0.77-1.82)$ & 0.433 \\
\hline Adequate & 620 & $117(18.9)$ & $1.35(0.93-1.97)$ & 0.117 & $1.34(0.92-1.96)$ & 0.129 \\
\hline More than adequate & 300 & $44(14.7)$ & 1 & - & 1 & - \\
\hline Excessive & 158 & $32(20.3)$ & $1.48(0.89-2.44)$ & 0.128 & $1.43(0.86-2.38)$ & 0.166 \\
\hline
\end{tabular}

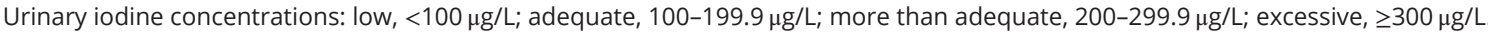

*Confounding factors in the multiple logistic regression analysis included age, sex, BMI, smoking, diabetes and hypertension.

$\mathrm{OR}$, odds ratio.

shown in Table 3 . All 22 studies used cross-sectional design, and sample size ranged from 209 to 15,008, with a total of 69,987 participants (Table 3). Among these 22 articles, most were conducted in Asians $(n=17)$, and the other five studies were from Europe (Table 3). Five studies focused on the association of iodine intake with TAI in children, and the other studies mainly assessed the relationship in adults (Table 3). Only three studies reported adjusted risk estimates and had low risk of confounding bias, but the other studies did not exclude the impact of confounding factors such as age and gender, which were considered at higher risk of confounding bias (Supplementary Table 4). All studies had low risk of information bias, and most studies had low risk of selection bias (Supplementary Table 4). According to the NOS criteria, most studies scored more than five points and thus had good quality, but three studies scored five or less and were considered to have suboptimal quality (Supplementary Table 4).

Because our preliminary pooled results suggested an obvious difference in the association of iodine intake with TAI between children and adults, we performed the doseresponse meta-analysis in adults and children separately. Dose-response meta-analysis of those 17 studies in adults revealed an obvious $U$-shaped association between iodine intake and TAI in adults with the lowest TAI risk at the UIC of $300 \mu \mathrm{g} / \mathrm{L}\left(P_{\text {Nonlinearity }}<0.0001\right.$, Fig. 1$)$, and both low iodine status and excessive iodine status could increase TAI risk in adults. The U-shaped curve between iodine intake and TAI in adults when the adequate iodine status (UIC 100-199.9 $\mu \mathrm{g} / \mathrm{L}$ ) was used as reference (Supplementary Fig. 2).
Dose-response meta-analysis of three studies with adjusted risk estimates and low risk of confounding bias also identified an U-shaped association between iodine intake and TAI in adults with the lowest TAI risk at the UIC of $250 \mu \mathrm{g} / \mathrm{L}\left(P_{\text {Nonlinearity }}<0.0001\right.$, Fig. 2). Dose-response meta-analysis of 14 studies without adjusted risk estimates and high risk of confounding bias further identified an U-shaped association between iodine intake and TAI risk in adults $\left(P_{\text {Nonlinearity }}<0.0001\right.$, Supplementary Fig. 3). After excluding studies with moderate risk or high risk of selection bias, the U-shaped association between iodine intake and TAI risk in adults still existed ( $P_{\text {Nonlinearity }}<0.0001$, Supplementary Fig. 4). Moreover, dose-response meta-analysis of 14 studies with good quality according to NOS criteria also revealed an U-shaped association between iodine intake and TAI in adults ( $P_{\text {Nonlinearity }}<0.0001$, Supplementary Fig. 5).

Dose-response meta-analysis of 13 studies on TPOAb positivity in adults also suggested an U-shaped association between iodine intake and TPOAb positivity in adults $\left(P_{\text {Nonlinearity }}=0.007\right)$. Dose-response meta-analysis of 12 studies on TGAb positivity further proved an obvious U-shaped association between iodine intake and TGAb positivity in adults $\left(P_{\text {Nonlinearity }}<0.0001\right)$.

There were only five studies assessing the association between iodine intake and TAI in children. Doseresponse meta-analysis of those five studies suggested a nonlinear association between iodine intake and TAI in children with the lowest TAI risk at the UIC of $50 \mu \mathrm{g} / \mathrm{L}$ $\left(P_{\text {Nonlinearity }}=0.022\right)$, but the U-shaped association was not observed (Supplementary Fig. 6). When the UIC 


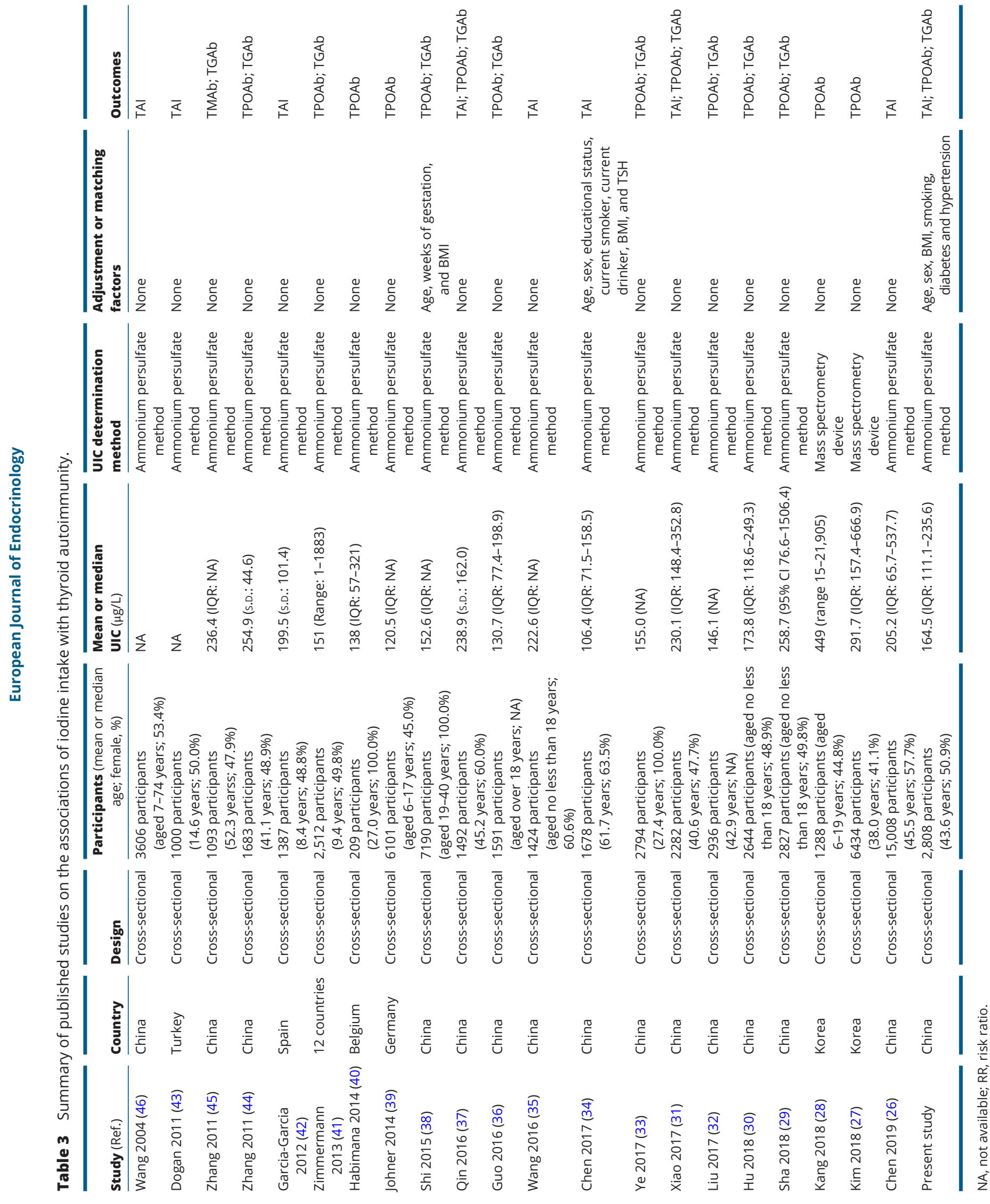




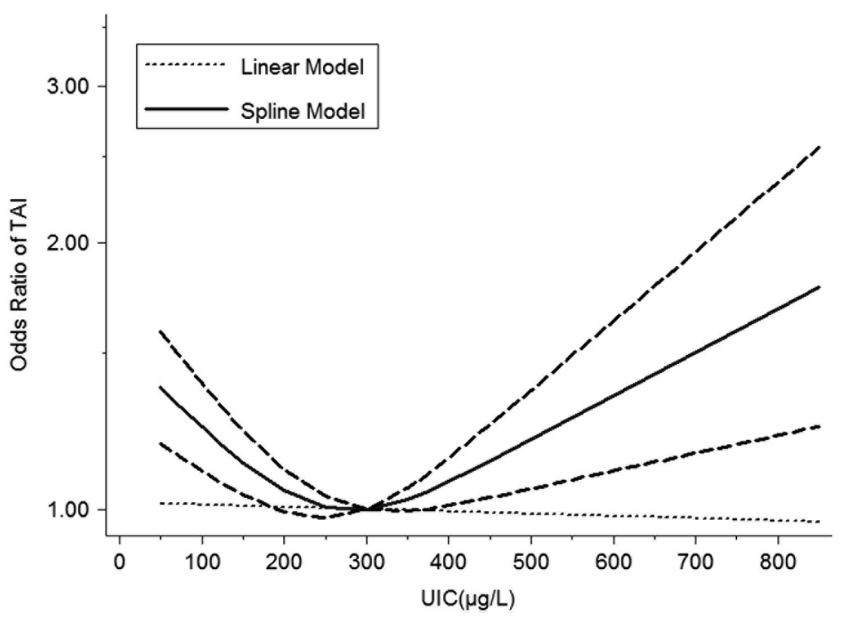

Figure 1

U-shaped association between iodine intake and thyroid autoimmunity in adults in the dose-response meta-analysis of 17 studies in adults. The reference level of UIC is $300 \mu \mathrm{g} / \mathrm{L}$. The black solid line represents the odds ratio and the long black dash line represents 95\% confidence intervals of the fitted nonlinear trend. The short dash line represents the linear trend. The spline model was the optimal model, while the curve for the linear model was shown to display the difference in the patterns between the spline model and the linear model.

increased from $50 \mu \mathrm{g} / \mathrm{L}$ to $250 \mu \mathrm{g} / \mathrm{L}$, the risk of TAI in children gradually increased. However, risk of TAI in children no longer increased when UIC was over $250 \mu \mathrm{g} / \mathrm{L}$ (Supplementary Fig. 6).

\section{Discussion}

Iodine intake has long been regarded as an environmental determinant for thyroid dysfunction, but its relationship with TAI is still controversial. Additionally, most previous studies did not adjust the influence of other confounding factors such as gender and age, and thus, were unable to evaluate the independent impact of iodine status on TAI risk. Our cross-sectional study adjusted the influence of confounding factors through multivariate logistic regression analysis, and thus was able to evaluate the independent role of iodine status on TAI risk. Additionally, we also performed a dose-response meta-analysis of 22 epidemiological studies, which was the first dose-response meta-analysis investigating the impact of iodine status on TAI risk. The cross-sectional study showed an U-shaped relationship between iodine intake and TAI in adults.

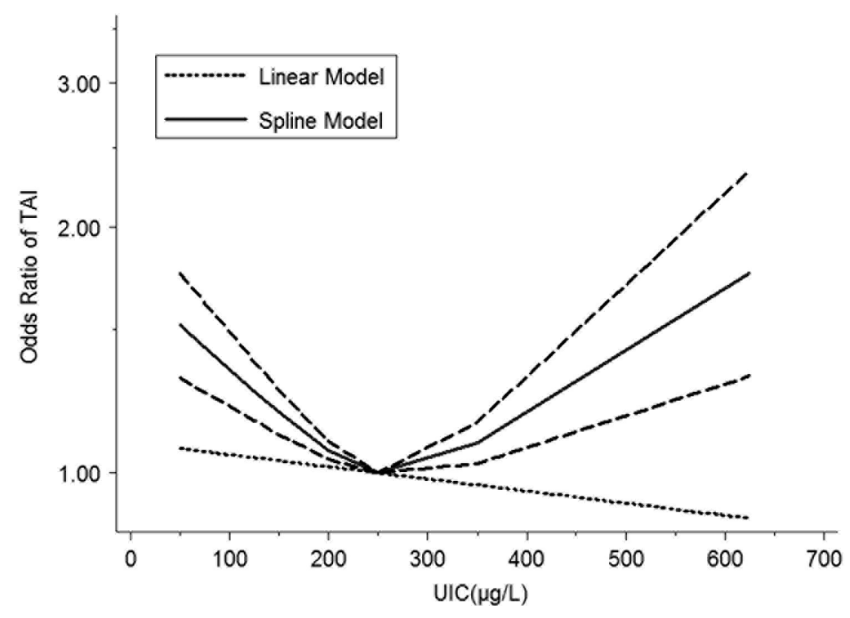

Figure 2

U-shaped association between iodine intake and thyroid autoimmunity in adults in the dose-response meta-analysis of three studies in adults with low risk of confounding bias. The reference level of UIC is $250 \mu \mathrm{g} / \mathrm{L}$. The black solid line represents the odds ratio and the long black dash line represents $95 \%$ confidence intervals of the fitted nonlinear trend. The short dash line represents the linear trend. The spline model was the optimal model, while the curve for the linear model was shown to display the difference in the patterns between the spline model and the linear model.

The dose-response meta-analysis further validated the U-shaped relationship between iodine intake and TAI in adults, which proved the significantly increased risk of TAI among individuals with either iodine deficiency or excessive iodine status. Therefore, the study provides strong epidemiological evidence for the U-shaped relationship between iodine intake and TAI in adults, and both iodine deficiency and iodine excess are risk factors of TAI.

The consequence of iodine status on human health has gained rapidly growing concerns in the past decade, and iodine status has long been regarded as a key determinant of thyroid disorders in adults $(17,47)$. World Health Organization (WHO) suggests that an adequate UIC should be between 100 and $200 \mu \mathrm{g} / \mathrm{L}$, and an UIC in the range of $200-299 \mu \mathrm{g} / \mathrm{L}$ and $>300 \mu \mathrm{g} / \mathrm{L}$ indicate more than adequate intake and the presence of excessive iodine, respectively (21). According to the criteria of iodine status above, there are a large percentage of persons with either iodine deficiency or excessive iodine $(28,48,49)$. It has been clear that iodine deficiency can result in impaired production of thyroid hormones and thus cause adverse effects on human health. Iodine deficiency can induce 
a number of iodine deficiency disorders (IDDs), such as hypothyroidism and goiter (50). Many countries have implemented universal salt iodization (USI) to eliminate IDDs, and iodine prophylaxis interventions have improved iodine status and reduced the prevalence of IDDs worldwide $(51,52)$.

Though iodine supplementation is essential in preventing IDDs, the adverse impact of iodine excess intake has to be taken into account. Currently, it remains a medical challenge to optimize iodine intake (53). Some studies also have shown an adverse impact of USI on the prevalence of other thyroid diseases such as hyperthyroidism (54). Therefore, a careful monitoring of iodine status is required to optimize USI and decrease the risk of thyroid disorders $(55,56)$. Additionally, though numerous studies have been performed to define the impact of iodine intake on various thyroid diseases such as thyroid cancer, TAI and hypothyroidism, no definite conclusion is available (17). Current studies suggest that the impact of iodine on different types of thyroid diseases is likely inconsistent, and the acceptable UIC range may thus vary in monitoring iodine supplementation across different types of thyroid diseases (17).

Similar to other thyroid diseases, optimization of iodine intake is also critical for the effective reduction of TAI prevalence. The classification method for iodine nutrition from WHO is mainly based on the relationship between UIC and IDDs such as hypothyroidism and thyroid goiter, in which the recommended UIC for the prevention of IDDs is within 100-199.9 $\mu \mathrm{g} / \mathrm{L}$ (21). TAI is an autoimmune disease occurring in thyroid and has an obviously distinct pathogenic mechanism when compared with IDDs. Our study suggests an optimal iodine intake of UIC between 200 and $300 \mu \mathrm{g} / \mathrm{L}$ for the prevention of TAI, while those with UIC either less than 200 or more than $400 \mu \mathrm{g} / \mathrm{L}$ are at increased risk of TAI. Therefore, iodine supplementation or salt iodization are recommended for individuals with iodine deficiency to reduce TAI risk, but avoiding excessive intake is also essential to reduce TAI risk. Additionally, because either iodine excess status or iodine deficiency can increase TAI risk, the monitoring of iodine status is essential to improve the benefits of iodine supplementation and minimize its adverse influence by avoiding excessive intake.

The association between iodine intake and TAI is still controversial in children. An epidemiological study by Tsatsoulis et al. reported that children with TAI had obviously higher UIC than Non-TAI controls (57). A large cross-sectional study by Zimmermann et al. revealed that TAI risk was not increased by either iodine deficiency or iodine excess in children (41). The current dose-response meta-analysis included only five studies assessing the association between iodine intake and TAI in children, and showed a nonlinear association between iodine intake and TAI in children with the lowest TAI risk at the UIC of $50 \mu \mathrm{g} / \mathrm{L}$ (Supplementary Fig. 6), which was different from the U-shaped relationship in adults. However, owing to the limited number of eligible studies in children, our study was still unable to establish a definite relationship between iodine intake and TAI risk in children. Considering the critical significance of iodine status in children, future studies are recommended to define the impact of iodine status on TAI risk in children.

As shown in the Supplementary table 4, confounding bias is a major threat to the validity of the pooled outcomes in our study. Both age and gender have long been regarded as important impact factors for both UIC and TAI, and they may cause the risk of confounding bias. However, most included studies did not adjust for age and gender, which increased the risk of confounding bias. Considering the limited number of studies with low risk of confounding risk, the findings in our study still need to be validated in more future studies with adequate adjustment for confounding factors. Besides, our cross-sectional study suggested that participants with high UIC had lower BMI, lower prevalence of diabetes and lower prevalence of hypertension than those with lower UIC (Table 1), suggesting that diabetes, BMI and hypertension were possible confounding factors. Therefore, to adequately assess the relationship between iodine and TAI risk, future studies need adjusting for all possible confounding factors through multivariate logistic regression analysis.

The mechanism of TAI is still largely elusive. $\mathrm{T}$ cells and $\mathrm{B}$ cells are the main parts of adaptive immune system which are considered to exert critical roles in the development of TAI, but recent studies indicate that innate immunity is also an essential part in the etiology of TAI $(1,58,59)$. Similar to other autoimmune diseases, TAI is preceded by an absence of immune balance among immune cells such as T cells, B cells and macrophages (58). Previous studies have established that both genetic and environmental risk factors are involved in the etiology of TAI, both of which may initiate the autoimmune attacks to thyroid antigens by breaking up the immune balance among immune cells (58). Though high iodine has been identified as an important risk factor of TAI, the underlying molecular mechanisms have not yet been sufficiently clarified. Some studies proposed several possible explanations below. Firstly, excessive iodine intake could increase thyroglobulin immunogenicity and 
TAI risk by unmasking a cryptic epitope on thyroglobulin, which could initiate the autoimmune process during TAI development (60). Additionally, the direct cytotoxic injury of thyroid cells caused by iodine has been well defined, which is an important event underlying the increased risk of TAI among individuals with excessive iodine intake (61, $62,63)$. Finally, the direct stimulatory effect of iodine on immune cells may also be involved in the development of TAI. For instance, iodine could promote IgG synthesis in human peripheral blood lymphocytes in vitro, which indicated that iodine may enhance antibody synthesis by immune cells (64). However, the molecular mechanisms for the stimulatory effect of iodine on immune cells are largely not explored, which need to be elaborated in future studies.

The findings from the current study reveal iodine deficiency as a risk factor for TAI. The finding was supported by one previous study by Mooij et al. which reported that iodine deficiency could induce thyroid autoimmune reactivity and increased intrathyroidal dendritic cells, $\mathrm{CD}^{+} \mathrm{T}$ cells and $\mathrm{CD} 8^{+} \mathrm{T}$ cells in Wistar rats (65). There are several possible explanations for the increased risk of TAI in individuals with iodine deficiency. Firstly, since patients with thyroid nodular goiter often have concurrent thyroid antibodies, iodine deficiency thus may indirectly increase the TAI prevalence by increasing the prevalence of thyroid nodular goiter and causing damages to thyroid tissues (17, $66,67,68,69)$. In addition, several studies have found that iodine can contribute to redox balance and iodine deficiency increases the degree of oxidative stress which may further promote immune response and increase autoimmune attack $(70,71,72)$. Finally, TSH level is intensively related to iodine status, and iodine deficiency can result in increased TSH level $(17,73)$. Some studies found that TSH have an important role in regulating both the function of the immune system and immune response $(74,75,76,77,78,79,80,81)$. Therefore, the increased TSH level among hypothyroidism individuals caused by iodine deficiency may induce aberrant immune response and further promote the development of TAI. However, the mechanisms underlying the increased risk of TAI among individuals with iodine deficiency are still largely unknown and need to be elucidated in more future studies.

There were several limitations in our study. Firstly, there was obvious difference in the assays or cutoffs of thyroid antibodies in those studies, which may result in the difference in the reported associations of iodine intake with thyroid antibodies. Secondly, we only assessed the status of TPOAb and TGAb by a cross-sectional study of nearly 3000 participants and did not evaluate the impact of iodine intake on risk of thyroid-stimulating hormone receptor antibodies (TRAb) positivity. The prevalence of TRAb is relatively low, and a study with a larger number of participants is necessary to adequately evaluate the impact of iodine intake on risk of TRAb positivity. Thirdly, most included studies used cross-sectional study design and thus had limited ability to assess the causality relationship between iodine and TAI. The relationship between iodine intake and TAI found in this study still needs to be validated in more prospective cohort studies or clinical controlled trials. Fourthly, apart from iodine status, there are also several other factors affecting TAI risk, such as gender, age and smoking. However, the interactions of iodine with other risk factors in the development of TAI are so far poorly defined, and future studies are needed to uncover them. Fifthly, most included studies were performed in Asian populations, and the findings may be unable to be generalized to other populations, such as Caucasians and Africans. More studies from Caucasians and Africans are needed to validate the relationship between iodine intake and TAI risk. Finally, there is a high possibility for iodine-gene interactions in the development of TAI. However, the iodine-gene interaction in the development of TAI is so far largely elusive. For instance, studies on the relationship between iodine status and TAI risk among individuals with predisposing genetic factors are still lacking, and further studies are warranted.

In conclusion, the findings in our study provide convincing evidence for the U-shaped relationship between iodine intake and TAI risk in adults, and both iodine deficiency and excessive iodine status are risk factors of TAI among adults. The findings have important clinical implications for iodine interventions, and monitoring iodine status is essential for an optimal iodine status. However, uncertainty remains about the relationship between iodine intake and TAI in specific populations such as children and those with predisposing genetic factors or family history, and it requires evidence from more longitudinal studies. Moreover, the pathogenic mechanisms underlying the roles of iodine in TAI are still largely elusive, and more studies are warranted to better elucidate those mechanisms.

\section{Supplementary data}

This is linked to the online version of the paper at https://doi.org/10.1530/ EJE-19-0212.

Declaration of interest

The authors declare that there is no conflict of interest that could be perceived as prejudicing the impartiality of this study. 


\section{Funding}

The present work was supported by grants from the National Natural Science Foundation of China (Grant No. 81873636 and 81670722) and The Research Fund for Public Welfare, National Health and Family Planning Commission of China (Grant No. 201402005).

\section{References}

1 Antonelli A, Ferrari SM, Corrado A, Di Domenicantonio A \& Fallahi P. Autoimmune thyroid disorders. Autoimmunity Reviews 2015 14 174-180. (https://doi.org/10.1016/j.autrev.2014.10.016)

2 Derakhshan A, Korevaar TIM, Taylor PN, Levie D, Guxens M, Jaddoe VWV, Nelson SM, Tiemeier H \& Peeters RP. The association of maternal thyroid autoimmunity during pregnancy with child IQ. Journal of Clinical Endocrinology and Metabolism 2018103 3729-3736. (https://doi.org/10.1210/jc.2018-00743)

3 De Leo $S$ \& Pearce EN. Autoimmune thyroid disease during pregnancy. Lancet: Diabetes and Endocrinology 20186 575-586. (https://doi.org/10.1016/S2213-8587(17)30402-3)

4 Hollowell JG, Staehling NW, Flanders WD, Hannon WH, Gunter EW, Spencer CA \& Braverman LE. Serum TSH, T(4), and thyroid antibodies in the United States population (1988 to 1994). National health and nutrition examination survey (NHANES III). Journal of Clinical Endocrinology and Metabolism 200287 489-499. (https://doi. org/10.1210/jcem.87.2.8182)

5 Nagayama Y. Thyroid autoimmunity and thyroid cancer - the pathogenic connection: a 2018 update. Hormone and Metabolic Research 201850 922-931. (https://doi.org/10.1055/a-0648-4593)

6 Frohlich E \& Wahl R. Thyroid autoimmunity: role of anti-thyroid antibodies in thyroid and extra-thyroidal diseases. Frontiers in Immunology 20178 521. (https://doi.org/10.3389/fimmu.2017.00521)

7 Boi F, Pani F \& Mariotti S. Thyroid autoimmunity and thyroid cancer: review focused on cytological studies. European Thyroid Journal 20176 178-186. (https://doi.org/10.1159/000468928)

8 Chen WH, Chen YK, Lin CL, Yeh JH \& Kao CH. Hashimoto's thyroiditis, risk of coronary heart disease, and L-thyroxine treatment: a nationwide cohort study. Journal of Clinical Endocrinology and Metabolism 2015100 109-114. (https://doi.org/10.1210/jc.20142990)

9 Zoller B, Li X, Sundquist J \& Sundquist K. Risk of subsequent coronary heart disease in patients hospitalized for immune-mediated diseases: a nationwide follow-up study from Sweden. PLoS ONE 2012 7 e33442. (https://doi.org/10.1371/journal.pone.0033442)

10 Ferrari SM, Fallahi P, Ruffilli I, Elia G, Ragusa F, Benvenga S \& Antonelli A. The association of other autoimmune diseases in patients with Graves' disease (with or without ophthalmopathy): review of the literature and report of a large series. Autoimmunity Reviews 201918 287-292. (https://doi.org/10.1016/j. autrev.2018.10.001)

11 Liu H, Shan Z, Li C, Mao J, Xie X, Wang W, Fan C, Wang H, Zhang H, Han C et al. Maternal subclinical hypothyroidism, thyroid autoimmunity, and the risk of miscarriage: a prospective cohort study. Thyroid 201424 1642-1649. (https://doi.org/10.1089/ thy.2014.0029)

12 Smith MJ, Rihanek M, Coleman BM, Gottlieb PA, Sarapura VD \& Cambier JC. Activation of thyroid antigen-reactive B cells in recent onset autoimmune thyroid disease patients. Journal of Autoimmunity 201889 82-89. (https://doi.org/10.1016/j.jaut.2017.12.001)

13 Jonsdottir B, Lundgren M, Wallengren S, Lernmark Å, Jonsson I, Elding Larsson H \& DiPiS Study Group. Are perinatal events risk factors for childhood thyroid autoimmunity? European Thyroid Journal 20176 298-306. (https://doi.org/10.1159/000479964)

14 Bliddal S, Nielsen CH \& Feldt-Rasmussen U. Recent advances in understanding autoimmune thyroid disease: the tallest tree in the forest of polyautoimmunity. F1000Research 20176 1776. (https://doi. org/10.12688/f1000research.11535.1)

15 Ambroziak U, Hybsier S, Shahnazaryan U, Krasnodebska-Kiljanska M, Rijntjes E, Bartoszewicz Z, Bednarczuk T \& Schomburg L. Severe selenium deficits in pregnant women irrespective of autoimmune thyroid disease in an area with marginal selenium intake. Journal of Trace Elements in Medicine and Biology 201744 186-191. (https://doi. org/10.1016/j.jtemb.2017.08.005)

16 Kim M, Song E, Oh HS, Park S, Kwon H, Jeon MJ, Kim WG, Kim WB, Shong YK \& Kim TY. Vitamin D deficiency affects thyroid autoimmunity and dysfunction in iodine-replete area: Korea national health and nutrition examination survey. Endocrine 201758 332-339. (https://doi.org/10.1007/s12020-017-1425-z)

17 Zimmermann MB \& Boelaert K. Iodine deficiency and thyroid disorders. Lancet: Diabetes and Endocrinology 20153 286-295. (https:// doi.org/10.1016/S2213-8587(14)70225-6)

18 Taylor PN, Albrecht D, Scholz A, Gutierrez-Buey G, Lazarus JH, Dayan CM \& Okosieme OE. Global epidemiology of hyperthyroidism and hypothyroidism. Nature Reviews: Endocrinology 201814 301-316. (https://doi.org/10.1038/nrendo.2018.18)

19 Kahaly GJ, Dienes HP, Beyer J \& Hommel G. Iodide induces thyroid autoimmunity in patients with endemic goitre: a randomised, double-blind, placebo-controlled trial. European Journal of Endocrinology 1998139 290-297. (https://doi.org/10.1530/ eje.0.1390290)

20 von Elm E, Altman DG, Egger M, Pocock SJ, Gøtzsche PC \& Vandenbroucke JP \& STROBE Initiative. The Strengthening the Reporting of Observational Studies in Epidemiology (STROBE) statement: guidelines for reporting observational studies. PLoS Medicine 20074 e296. (https://doi.org/10.1371/journal. pmed.0040296)

21 WHO. Assessment of Iodine Deficiency Disorders and Monitoring Their Elimination - A Guide for Programme Managers, 3rd ed. Geneva, Switzerland: WHO, 2008.

22 Dekkers OM, Vandenbroucke JP, Cevallos M, Renehan AG, Altman DG, Egger M. COSMOS-E: guidance on conducting systematic reviews and meta-analyses of observational studies of etiology. PLoS Medicine 201916 e1002742. (https://doi.org/10.1371/ journal.pmed.1002742)

23 Orsini N, Li R, Wolk A, Khudyakov P \& Spiegelman D. Metaanalysis for linear and nonlinear dose-response relations: examples, an evaluation of approximations, and software. American Journal of Epidemiology 2012175 66-73. (https://doi. org/10.1093/aje/kwr265)

24 Orsini N, Bellocco R \& Greenland S. Generalized least squares for trend estimation of summarized dose-response data. STATA Journal: Promoting Communications on Statistics and Stata 20066 40-57. (https://doi.org/10.1177/1536867X0600600103)

25 DerSimonian R \& Laird N. Meta-analysis in clinical trials revisited. Contemporary Clinical Trials 201545 139-145. (https://doi. org/10.1016/j.cct.2015.09.002)

26 Chen X, Chen L, Lian X, Liu C, Shan Z, Shi B, Shi L, Tong N, Weng J, Zhao $\mathrm{J}$ et al. Urinary iodine concentration is inversely associated with the thyroglobulin antibody. Endocrine Practice 201925 454-460. (https://doi.org/10.4158/EP-2018-0252)

27 Kim HI, Oh HK, Park SY, Jang HW, Shin MH, Han JM, Bae JC, Kim SW, Kim TH \& Chung JH. Non-immune-related hypothyroidism and its relationship with excess iodine. European Journal of Nutrition 2018 [epub]. (https://doi.org/10.1007/s00394-018-1837-4)

28 Kang MJ, Hwang IT \& Chung HR. Excessive iodine intake and subclinical hypothyroidism in children and adolescents aged 6-19 years: results of the sixth Korean national health and nutrition examination survey, 2013-2015. Thyroid 201828 773-779. (https:// doi.org/10.1089/thy.2017.0507)

29 Sha L, Wang T, Lu Q, Zhang H, Xue H, Wang J, Zhao W, Li D, Lei H $\&$ He L. Study on the correlation between iodine nutrition level 
and thyroid diseases in some areas of Ningxia. Chinese Journal of Endocrinology and Metabolism 201834 394-397.

$30 \mathrm{Hu}$ W, Zeng Z \& Zhang H. Epidemiological investigation of iodine nutrient status and the relationship between iodized salt and thyroid disease in Hunan province. Journal of Chinese Practical Diagnosis and Therapy 201832 440-444.

31 Xiao Y \& Qin G. Epidemiological study on the relationship between iodine nutrition and thyroid diseases of Han population in Henan province. Masters Thesis. Zhengzhou University, 2017.

32 Liu X \& Fan Y. Investigation on situations of iodine nutritional, thyroid disease and some impacting factors in Uighur residents in Urumqi and Toksun. Masters Thesis. Xinjiang Medical University, 2017.

33 Ye W, He L, Liu H, Hua L, Lin C \& Luo Y. Iodine nutrition and thyroid function in women with early pregnancy in Panyu, Guangzhou. Guangdong Medical Journal 201738 1865-1867.

34 Chen C, Xu H, Chen Y, Chen Y, Li Q, Hu J, Liang W, Cheng J, Xia F, Wang $\mathrm{C}$ et al. Iodized salt intake and its association with urinary iodine, thyroid peroxidase antibodies, and thyroglobulin antibodies among urban Chinese. Thyroid 201727 1566-1573. (https://doi. org/10.1089/thy.2017.0385)

35 Wang JJ \& Sun H. A cross-sectional survey on iodine nutrition, the thyroid morphology and its function of Wansong residents in Wuhan. Masters Thesis. Huazhong University of Science and Technology, 2016.

36 Guo Y, Zynat J, Xu Z, Wang X, Osiman R, Zhao H, Tuhuti A, Abdunaimu $\mathrm{M}$, Wang $\mathrm{H}$, Jin $\mathrm{X}$ et al. Iodine nutrition status and thyroid disorders: a cross-sectional study from the Xinjiang autonomous region of China. European Journal of Clinical Nutrition 201670 1332-1336. (https://doi.org/10.1038/ejcn.2016.82)

37 Qin C \& Zhang Q. Influence of iodine nutrient status on autoimmune thyroid disease in iodine excessive area. Journal of Guiyang Medical College 201641 194-196.

38 Shi X, Han C, Li C, Mao J, Wang W, Xie X, Li C, Xu B, Meng T, $\mathrm{Du} \mathrm{J}$ et al. Optimal and safe upper limits of iodine intake for early pregnancy in iodine-sufficient regions: a cross-sectional study of 7190 pregnant women in China. Journal of Clinical Endocrinology and Metabolism 2015100 1630-1638. (https://doi.org/10.1210/jc.20143704)

39 Johner SA, Thamm M, Stehle P, Nothlings U, Kriener E, Volzke H, Gartner R \& Remer T. Interrelations between thyrotropin levels and iodine status in thyroid-healthy children. Thyroid $2014 \mathbf{2 4}$ 1071-1079. (https://doi.org/10.1089/thy.2013.0480)

40 Habimana L, Twite KE, Daumerie C, Wallemacq P, Donnen P, Kalenga MK \& Robert A. High prevalence of thyroid dysfunction among pregnant women in Lubumbashi, Democratic Republic of Congo. Thyroid 201424 568-575. (https://doi.org/10.1089/ thy.2013.0261)

41 Zimmermann MB, Aeberli I, Andersson M, Assey V, Yorg JA, Jooste P, Jukic T, Kartono D, Kusic Z, Pretell E et al. Thyroglobulin is a sensitive measure of both deficient and excess iodine intakes in children and indicates no adverse effects on thyroid function in the UIC range of 100-299 mug/L: a UNICEF/ICCIDD study group report. Journal of Clinical Endocrinology and Metabolism 201398 1271-1280. (https:// doi.org/10.1210/jc.2012-3952)

42 Garcia-Garcia E, Vazquez-Lopez MÁ, Garcia-Fuentes E, RodriguezSanchez FI, Munoz FJ, Bonillo-Perales A \& Soriguer F. Iodine intake and prevalence of thyroid autoimmunity and autoimmune thyroiditis in children and adolescents aged between 1 and 16 years. European Journal of Endocrinology 2012167 387-392. (https://doi. org/10.1530/EJE-12-0267)

43 Dogan M, Acikgoz E, Acikgoz M, Cesur Y, Ariyuca S \& Bektas MS. The frequency of Hashimoto thyroiditis in children and the relationship between urinary iodine level and Hashimoto thyroiditis. Journal of Pediatric Endocrinology and Metabolism 201124 75-80.
44 Zhang M, Zhang L, Fang Y, Zeng X, Zhu J, Abulikemu \& Yiliduosi. Relationship between iodine intake and the prevalence of thyroid disease in Urumqi, Xinjiang. Chinese Journal of Endocrinology and Metabolism 201127 972-974.

45 Zhang MC, Zhang L, Fan Y, Zeng X, Zhu J, Abulidemu T \& Lilidusi A. Study of the relationship between iodine intake level and the occurrence of thyroid disease of Uygur nationality in Urumqi. Journal of Xinjiang Medical University 201134 1019-1021.

46 Wang YG, Yan S, Zhao S, Wang F \& Yu X. Correlation of Hashimoto's thyroiditis with urine iodine level among the coastal districts in Shandong province. Chinese Journal of Endocrinology and Metabolism $200420337-338$.

47 Monahan M, Boelaert K, Jolly K, Chan S, Barton P \& Roberts TE. Costs and benefits of iodine supplementation for pregnant women in a mildly to moderately iodine-deficient population: a modelling analysis. Lancet: Diabetes and Endocrinology 20153 715-722. (https:// doi.org/10.1016/S2213-8587(15)00212-0)

48 Vanderpump MP, Lazarus JH, Smyth PP, Laurberg P, Holder RL, Boelaert K, Franklyn JA \& British Thyroid Association UK Iodine Survey Group. Iodine status of UK schoolgirls: a cross-sectional survey. Lancet 2011377 2007-2012. (https://doi.org/10.1016/S01406736(11)60693-4)

49 Mioto VCB, Monteiro ACCNG, de Camargo RYA, Borel AR, Catarino RM, Kobayashi S, Chammas MC \& Marui S. High prevalence of iodine deficiency in pregnant women living in adequate iodine area. Endocrine Connections 20187 762-767. (https:// doi.org/10.1530/EC-18-0131)

50 Zimmermann MB, Jooste PL \& Pandav CS. Iodine-deficiency disorders. Lancet 2008372 1251-1262. (https://doi.org/10.1016/ S0140-6736(08)61005-3)

51 Pearce EN. The American Thyroid Association: statement on universal salt iodization. Thyroid 201727 137. (https://doi. org/10.1089/thy.2016.0678)

52 Dold S, Zimmermann MB, Jukic T, Kusic Z, Jia Q, Sang Z, Quirino A, San Luis TOL, Fingerhut R, Kupka R et al. Universal salt iodization provides sufficient dietary iodine to achieve adequate iodine nutrition during the first 1000 days: a cross-sectional multicenter study. Journal of Nutrition 2018148 587-598. (https://doi.org/10.1093/jn/nxy015)

53 Pearce EN, Andersson M \& Zimmermann MB. Global iodine nutrition: where do we stand in 2013? Thyroid 201323 523-528. (https://doi.org/10.1089/thy.2013.0128)

54 Shan Z, Chen L, Lian X, Liu C, Shi B, Shi L, Tong N, Wang S, Weng J, Zhao J et al. Iodine status and prevalence of thyroid disorders after introduction of mandatory universal salt iodization for 16 years in China: a cross-sectional study in 10 cities. Thyroid 201626 1125-1130. (https://doi.org/10.1089/thy.2015.0613)

55 The EUthyroid Consortium. The Krakow declaration on iodine: tasks and responsibilities for prevention programs targeting iodine deficiency disorders. European Thyroid Journal 20187 201-204. (https://doi.org/10.1159/000490143)

56 Volzke H, Caron P, Dahl L, de Castro JJ, Erlund I, Gaberscek S, Gunnarsdottir I, Hubalewska-Dydejczyk A, Ittermann T, Ivanova L et al. Ensuring effective prevention of iodine deficiency disorders. Thyroid 201626 189-196. (https://doi.org/10.1089/thy.2015.0543)

57 Tsatsoulis A, Johnson EO, Andricula M, Kalogera C, Svarna E, Spyroy P, Seferiadis K \& Tsolas $\mathrm{O}$. Thyroid autoimmunity is associated with higher urinary iodine concentrations in an iodinedeficient area of northwestern Greece. Thyroid 19999 279-283. (https://doi.org/10.1089/thy.1999.9.279)

58 McLachlan SM \& Rapoport B. Breaking tolerance to thyroid antigens: changing concepts in thyroid autoimmunity. Endocrine Reviews 2014 35 59-105. (https://doi.org/10.1210/er.2013-1055)

59 Smith TJ \& Hegedus L. Graves' disease. New England Journal of Medicine 2016375 1552-1565. (https://doi.org/10.1056/ NEJMra1510030) 
60 Latrofa F, Fiore E, Rago T, Antonangeli L, Montanelli L, Ricci D, Provenzale MA, Scutari M, Frigeri M, Tonacchera M et al. Iodine contributes to thyroid autoimmunity in humans by unmasking a cryptic epitope on thyroglobulin. Journal of Clinical Endocrinology and Metabolism 201398 E1768-E1774. (https://doi.org/10.1210/jc.20132912)

$61 \mathrm{Li} \mathrm{M} \&$ Boyages SC. Iodide induced lymphocytic thyroiditis in the $\mathrm{BB} / \mathrm{W}$ rat: evidence of direct toxic effects of iodide on thyroid subcellular structure. Autoimmunity 199418 31-40. (https://doi. org/10.3109/08916939409014677)

62 Bagchi N, Brown TR \& Sundick RS. Thyroid cell injury is an initial event in the induction of autoimmune thyroiditis by iodine in obese strain chickens. Endocrinology 1995136 5054-5060. (https://doi. org/10.1210/endo.136.11.7588241)

63 Jiang Y, Guo X, Sun Q, Shan Z \& Teng W. Effects of excess fluoride and iodide on thyroid function and morphology. Biological Trace Element Research 2016170 382-389. (https://doi.org/10.1007/s12011015-0479-0)

64 Weetman AP, McGregor AM, Campbell H, Lazarus JH, Ibbertson HK $\&$ Hall R. Iodide enhances IgG synthesis by human peripheral blood lymphocytes in vitro. Acta Endocrinologica 1983103 210-215. (https://doi.org/10.1530/acta.0.1030210)

65 Mooij P, de Wit HJ, Bloot AM, Wilders-Truschnig MM \& Drexhage HA. Iodine deficiency induces thyroid autoimmune reactivity in Wistar rats. Endocrinology 1993133 1197-1204. (https:// doi.org/10.1210/endo.133.3.8103449)

66 Giray B \& Hincal F. Oxidative DNA base damage, antioxidant enzyme activities and selenium status in highly iodine-deficient goitrous children. Free Radical Research 200236 55-62. (https://doi. org/10.1080/10715760210162)

67 Maier J, van Steeg H, van Oostrom C, Paschke R, Weiss RE \& Krohn K. Iodine deficiency activates antioxidant genes and causes DNA damage in the thyroid gland of rats and mice. Biochimica and Biophysica Acta 20071773 990-999. (https://doi.org/10.1016/j. bbamcr.2007.03.011)

68 Craps J, Wilvers C, Joris V, De Jongh B, Vanderstraeten J, Lobysheva I, Balligand JL, Sonveaux P, Gilon P, Many MC et al. Involvement of nitric oxide in iodine deficiency-induced microvascular remodeling in the thyroid gland: role of nitric oxide synthase 3 and ryanodine receptors. Endocrinology 2015156 707-720. (https://doi.org/10.1210/ en.2014-1729)

69 Gerard AC, Poncin S, Caetano B, Sonveaux P, Audinot JN, Feron O, Colin IM \& Soncin F. Iodine deficiency induces a thyroid stimulating hormone-independent early phase of microvascular reshaping in the thyroid. American Journal of Pathology 2008172 748-760. (https:// doi.org/10.2353/ajpath.2008.070841)

70 Vidal ZE, Rufino SC, Tlaxcalteco EH, Trejo CH, Campos RM, Meza MN, Rodriguez RC \& Arroyo-Helguera O. Oxidative stress increased in pregnant women with iodine deficiency. Biological Trace
Element Research 2014157 211-217. (https://doi.org/10.1007/s12011014-9898-6)

71 Cuellar-Rufino S, Navarro-Meza M, Garcia-Solis P, Xochihua-Rosas I \& Arroyo-Helguera O. Iodine levels are associated with oxidative stress and antioxidant status in pregnant women with hypertensive disease. Nutricion Hospitalaria 201734 661-666. (https://doi. org/10.20960/nh.460)

72 Kurku H, Gencer A, Pirgon O, Buyukinan M \& Aslan N. Increased oxidative stress parameters in children with moderate iodine deficiency. Journal of Pediatric Endocrinology and Metabolism 201629 1159-1164. (https://doi.org/10.1515/jpem-2016-0077)

73 Zimmermann MB, Aeberli I, Melse-Boonstra A, Grimci L, Bridson J, Chaouki N, Mbhenyane X \& Jooste PL. Iodine treatment in children with subclinical hypothyroidism due to chronic iodine deficiency decreases thyrotropin and C-peptide concentrations and improves the lipid profile. Thyroid 200919 1099-1104. (https://doi. org/10.1089/thy.2009.0001)

74 Klein JR. Novel splicing of immune system thyroid stimulating hormone beta-subunit-genetic regulation and biological importance. Frontiers in Endocrinology 201910 44. (https://doi.org/10.3389/ fendo.2019.00044)

75 Blalock JE, Johnson HM, Smith EM \& Torres BA. Enhancement of the in vitro antibody response by thyrotropin. Biochemical and Biophysical Research Communications 1984125 30-34. (https://doi. org/10.1016/s0006-291x(84)80329-0)

76 Frohlich E \& Wahl R. MECHANISMS IN ENDOCRINOLOGY: Impact of isolated TSH levels in and out of normal range on different tissues. European Journal of Endocrinology 2016174 R29-R41. (https://doi. org/10.1530/EJE-15-0713)

77 Gagnon A, Lochnan HA, Tran CS \& Sorisky A. Thyroid-stimulating hormone acutely increases monocyte gene expression in vivo. Neuro Endocrinology Letters 201637 121-123.

78 Mester T, Raychaudhuri N, Gillespie EF, Chen H, Smith TJ \& Douglas RS. CD40 expression in fibrocytes is induced by TSH: potential synergistic immune activation. PLOS ONE 201611 e0162994. (https://doi.org/10.1371/journal.pone.0162994)

79 van der Weerd K, van Hagen PM, Schrijver B, Heuvelmans SJ, Hofland LJ, Swagemakers SM, Bogers AJ, Dik WA, Visser TJ, van Dongen JJ et al. Thyrotropin acts as a T-cell developmental factor in mice and humans. Thyroid 201424 1051-1061. (https://doi. org/10.1089/thy.2013.0396)

80 Wang J \& Klein JR. Hormonal regulation of extrathymic gut T cell development: involvement of thyroid stimulating hormone. Cellular Immunology 1995161 299-302. (https://doi.org/10.1006/ cimm.1995.1040)

81 Jara EL, Munoz-Durango N, Llanos C, Fardella C, Gonzalez PA, Bueno SM, Kalergis AM \& Riedel CA. Modulating the function of the immune system by thyroid hormones and thyrotropin. Immunology Letters 2017184 76-83. (https://doi.org/10.1016/j.imlet.2017.02.010)

Received 21 March 2019

Revised version received 4 June 2019

Accepted 17 June 2019 